\title{
Monogenic PD in Brazil: a step towards precision medicine
}

\author{
DP monogênica no Brasil: um passo em direção à medicina de precisão
}

Thomas COURTIN', Alexis BRICE

\author{
'Sorbonne Université, Paris \\ Brain Institute - Institut du \\ Cerveau - ICM, INSERM, CNRS, \\ Département de Génétique, \\ Hôpital Pitié-Salpêtrière, APHP, \\ Paris, France. \\ Thomas Courtin (10 https://orcid. \\ org/0000-0002-0275-2498; \\ Alexis Brice (1) https://orcid. \\ org/0000-0002-0275-2498 0000- \\ 0002-0941-3990 \\ Correspondence: Alexis Brice; \\ Email :alexis.brice@icm- \\ institute.org. \\ Conflict of interest: There is no \\ conflict of interest to declare. \\ Authors' contribution: Both \\ authors contributed equally to \\ this work. \\ Received on June 01, 2021; \\ Accepted on June 01, 2021.

\section{(c) BY}

I n this issue of Arquivos de Neuro-Psiquiatria, Santos-Lobato and colleagues ${ }^{1}$ publish a systematic review of the literature related to the genetics of Parkinson's Disease (PD) in Brazil. It represents the first attempt to gather all the current knowledge on monogenic forms of PD in a large South American country.

It is now widely accepted that a substantial proportion of risk for PD is driven by genetics. So far, more than a dozen genes with dominant, recessive or X-linked inheritance have been linked to PD, mostly in familial and/or early onset forms ${ }^{2}$. Another fraction of PD heritability is driven by almost 100 genetic risk factors identified to date ${ }^{3}$.

This meta-analysis comprises 32 studies variably testing one or more of 2 autosomal dominant and 7 autosomal recessives genes, in patients from different regions of Brazil, identifying mutations in 5 of these genes. However, inclusion criteria and techniques used for mutation detection varied greatly among studies limiting comparisons. Nevertheless, data from LRRK2 and PRKN, the most widely tested and frequent genes could be analyzed in detail. LRRK2 pathogenic mutations, mostly the common G2019S mutation, affected 3.5\% of Brazilian patients, reaching $5 \%$ in familial forms. This is compatible with the figures found in European populations ${ }^{4}$ which constitute the majority of the Brazilian ancestry. However, LKKR2 G2019S prevalence varies greatly among populations, exceeding 10\% in Ashkenazy Jews and 1/3 in North African PD cases ${ }^{5,6}$. Therefore, it would be very interesting to evaluate precisely its frequency in Brazilians of African and Amerindian ancestry. PRKN mutations were observed in $8.3 \%$ of the cases tested, reaching $9.3 \%$ in PD patients with early onset (EOPD). Clearly, these values are not reflecting the prevalence of PRKN cases among PD cases in Brazil. They are inflated because of the enrichment of EOPD and familial cases in the series tested and because cases with a single heterozygous mutation were frequent (44\%). Indeed, the frequency of PRKN cases varies greatly according to the age at onset and decreases sharply after 40 (42.2\% before age 20, 29\% between 21 and 30, 13\% between 31 and 40, and 4.4\% between 41 and 60, no cases after 60$)^{7}$. In addition, cases with a single heterozygous mutation might have either another undetected mutation representing true PRKN cases or no additional representing false PRKN cases. Finally, mutations in GBA are the most frequent and strongest risk factor for PD which would have been interesting to analyze at the Brazilian population level.

This study is a good and timely starting point for exploring PD genetics in Brazil. First, identifying monogenic forms is crucial for appropriate genetic counselling. The risk of recurrence in descendants of PRKN cases is low if the spouse is not a relative. The $50 \%$ risk of an offspring of a G2019S carrier should be mitigated by the fact that the penetrance of this mutation is greatly reduced down to approximately $1 / 6$ in North African populations or $30 \%$ in Ashkenazy Jews ${ }^{5,6}$. Second, since specific treatments are now tested in several genetic forms (e.g. LRRK2 inhibitors in cases with the G2019S mutation), these studies provide trial ready cohorts for precision medicine. Third, Brazil offers populations from diverse ancestry, including African and Ameridian who belong to underrepresented ethnic groups in PD research. It will be mandatory to determine whether genetic information coming for patients of European ancestry are transferable and clinically useful to other ethnic groups. The involvement of ethnic-specific genes or their different frequency among ethnies might need to adapt genetic testing. Nevertheless, with the advent of next generation sequencing technologies and their 
constantly decreasing price these questions should be answered soon. Indeed these technologies allow not only to test simultaneously all the relevant genes but also to better capture the diversity of disease causing mutations.

The authors underline the need to study the genetic basis of PD in a larger and more diverse population of Brazil. This would increase genetic diagnosis accuracy and define etiological subtypes, critical steps for the implementation of precision medicine for PD. A step toward this end is the GP2 initiative ${ }^{8}$ which aims through mobilizing a worldwide community of researchers and participants, to generate and to analyze genetic data on an extremely large scale.

\section{REFERENCES}

1. Bruno L, Santos-Lobato VT. Genetics of Parkinson's disease in Brazil: a systematic review of monogenic forms. Arq Neuropsiquiatr. 2021;79(7):612-23. https://doi.org/10.1590/0004-282XANP-2020-0409

2. Lunati A, Lesage S, Brice A. The genetic landscape of Parkinson's disease. Rev Neurol (Paris). 2018;174(9):628-43. https://doi. org/10.1016/j.neurol.2018.08.004

3. Nalls MA, Blauwendraat C, Vallerga CL, et al. Identification of novel risk loci, causal insights, and heritable risk for Parkinson's disease: a meta-analysis of genome-wide association studies. Lancet Neurol. 2019;18(12):1091-1102. https://doi.org/10.1016/S14744422(19)30320-5

4. Nichols WC, Pankratz N, Hernandez D, et al. Genetic screening for a single common LRRK2 mutation in familial Parkinson's disease.
Lancet. 2005;365(9457):410-2. https://doi.org/10.1016/S01406736(05)17828-3

5. Lesage S, Dürr A, Tazir M, et al. LRRK2 G2019S as a cause of Parkinson's disease in North African Arabs. N Engl J Med. 2006;354(4):422-3. https://doi.org/10.1056/NEJMc055540

6. Thaler A, Ash E, Gan-Or Z, Orr-Urtreger A, Giladi N. The LRRK2 G2019S mutation as the cause of Parkinson's disease in Ashkenazi Jews. J Neural Transm (Vienna). 2009;116(11):1473-82. https://doi. org/10.1007/s00702-009-0303-0

7. Lesage S, Lunati A, Houot M, et al. Characterization of recessive Parkinson's disease in a large multicenter study. Ann Neurol. 2020;88: 843-50. https://doi.org/10.1002/ana.25787

8. Global Parkinson's Genetics Program. GP2: The Global Parkinson's Genetics Program. Mov Disord. 2021;36(4):842-51. https://doi. org/10.1002/mds.28494 